\title{
Surface-plasmon-polariton-induced suppressed transmission through ultrathin metal disk arrays
}

Xiao, Sanshui; Mortensen, Asger

Published in:

Optics Letters

Link to article, DOI:

10.1364/OL.36.000037

Publication date:

2011

Document Version

Publisher's PDF, also known as Version of record

Link back to DTU Orbit

Citation (APA):

Xiao, S., \& Mortensen, A. (2011). Surface-plasmon-polariton-induced suppressed transmission through ultrathin metal disk arrays. Optics Letters, 36(1), 37-39. https://doi.org/10.1364/OL.36.000037

\section{General rights}

Copyright and moral rights for the publications made accessible in the public portal are retained by the authors and/or other copyright owners and it is a condition of accessing publications that users recognise and abide by the legal requirements associated with these rights.

- Users may download and print one copy of any publication from the public portal for the purpose of private study or research.

- You may not further distribute the material or use it for any profit-making activity or commercial gain

- You may freely distribute the URL identifying the publication in the public portal

If you believe that this document breaches copyright please contact us providing details, and we will remove access to the work immediately and investigate your claim. 


\title{
Surface-plasmon-polariton-induced suppressed transmission through ultrathin metal disk arrays
}

\author{
Sanshui Xiao* and N. Asger Mortensen \\ DTU Fotonik-Department of Photonics Engineering, Technical University of Denmark, DK-280o Kongens Lyngby, Denmark \\ ${ }^{*}$ Corresponding author: saxi@fotonik.dtu.dk
}

Received September 16, 2010; revised November 10, 2010; accepted November 20, 2010; posted December 1, 2010 (Doc. ID 135268); published December 20, 2010

\begin{abstract}
We report surface-plasmon-polariton-induced suppressed transmission through two-dimensional arrays of isolated metal disks with a thickness comparable to optical skin depth of the metal. A transmittance dip of $-17.5 \mathrm{~dB}$ is achieved at the resonant wavelength of $1524 \mathrm{~nm}$, compared to $-12 \mathrm{~dB}$ for closed film. Coupling the light into the surface-plasmon polariton results in enhanced absorption, which is potentially interesting in solar cell applications. (C) 2010 Optical Society of America

OCIS codes: $\quad 050.0050,240.6680,260.3910,260.5740$.
\end{abstract}

It is well known that a high proportion of light can be transmitted using an ultrathin metal film whose thickness is comparable to its optical penetration depth. When the film is periodically modulated by subwavelength apertures, one intuitively expects that the ultrathin film could transmit even more light because less material is blocking the light. Surprisingly, however, by means of analytical and numerical calculations [1] ], it was recently predicted that the transmission can nevertheless be totally suppressed. This nontrivial phenomenon is the complete opposite of the widely known extraordinary optical transmission of light through periodically modulated optical thick metal films, which was first reported by Ebbesen et al. [2]. Subsequently, the suppressed transmission of light through ultrathin films pierced with periodic arrays of subwavelength apertures was demonstrated experimentally[3,4]. Numerical calculations [5] also pointed out that near-zero transmission can be realized by a two-dimensional (2D) array of isolated metal disks, while an ultrathin metal film modulated by a 2D subwavelength hole array does not support a near-zero transmission band. In this Letter, we experimentally demonstrate near-zero transmission of light through $2 \mathrm{D}$ arrays of isolated metal disks with a thickness comparable to their optical skin depth. The suppressed transmission is associated with the resonance anomaly (one of Wood's anomalies [6-8]), which is attributed to the excitation of the surface-plasmon-polariton (SPP) resonance.

Figure 1(a) illustrates a 2D array of isolated gold circular disks on a quartz substrate, where the thickness, radius, and period of the disks are denoted by $t, r$, and $p$, respectively. The inset in Fig. 1(a) indicates the propagation direction and polarization of the incident plane wave. Here we suppose that a plane wave illuminates the structure at normal incidence. In our studies, we prepared $2 \mathrm{~cm} \times 2 \mathrm{~cm}^{2}$ samples with the standard electron beam lithography (EBL) technique. First, a $100 \mathrm{~nm}$ ZEP520A (3.6\%, Zeon Corp., Tokyo, Japan) resist was spin coated onto a $1 \mathrm{~mm}$ quartz substrate. Then a $15 \mathrm{~nm}$ aluminum layer was thermally deposited on top of the ZEP layer to prevent charging during EBL. The EBL exposure was done with a $100 \mathrm{kV}$ JEOL JBX-9300FS EBL tool $\left(200 \mu \mathrm{C} / \mathrm{cm}^{2}\right.$ dose, $2 \mathrm{nA}$ current, $6 \mathrm{~nm}$ spot size).
The aluminum layer was removed in undiluted MF-322 (Rohm and Haas, Coventry, UK), and the positive ZEP resist was developed in ZED-N50 (Zeon Corp.) developer. Then a brief descum process was carried out to remove any residual resist. Finally, $5 \mathrm{~nm}$ titanium and $15 \mathrm{~nm}$ gold were deposited before liftoff was performed, concluding the fabrication process. The scanning electron microscope image is shown in Fig. 1(b), where $t=15 \mathrm{~nm}, r=262.5 \mathrm{~nm}$, and $p=700 \mathrm{~nm}$. The fabricated samples were characterized in a free space measurement setup using a supercontinuum light source (500-1750 nm, SuperK SCB-Compact 100-PC), as illustrated in Fig. 2(a). First, the emitted beam from the light source was collimated and broadened via a parabolic mirror. Then the light was linearly polarized by a polarizer before reaching the sample. The transmitted light was reflected by a mirror and focused by another parabolic mirror, then finally collected by a fiber leading to a spectrum analyzer (400-1750 nm, Ando AQ-6315E).

The measured transmittances at normal incidence for different-sized structures are shown in Fig. 2(b), where the dashed curve illustrates the transmittance spectrum through a metal film with the same thickness as that of the gold disks. As represented in Fig. 2(b), one can observe that the transmission is significantly suppressed, especially for the case with $p=700 \mathrm{~nm}$ and $r=285.0 \mathrm{~nm}$.

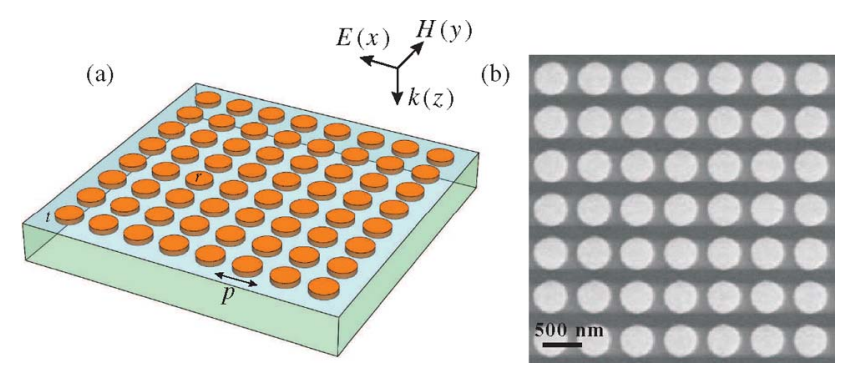

Fig. 1. (Color online) (a) Schematic illustration of a twodimensional periodic array of metal disks on a quartz wafer, where the thickness, radius of the disks, and period are denoted by $t, r$, and $p$, respectively. Axis shows the propagation direction and polarization of the incident plane wave. (b) Scanning electron microscope image of the structure, where $t=15 \mathrm{~nm}$, $r=262.5 \mathrm{~nm}$, and $p=700 \mathrm{~nm}$. 
The transmittance is almost totally suppressed, around $-17.5 \mathrm{~dB}$, at the resonant wavelength $\lambda_{0}=1524 \mathrm{~nm}$. Compared to the case of unstructured metal film of the same thickness (shown by the dashed curve), the transmittance drops from -12 to $-17.5 \mathrm{~dB}$. Note that in this case the metal surface coverage is only around $52 \%$. The result shows a phenomenon opposite to the extraordinary optical transmission of light through film that is perforated by subwavelength holes [9-12]. Intuitively, one expects that the metal disk array, compared with the closed film, will transmit more light. However, we emphasize that the measurement demonstrates less light transmitted through the array of gold disks. When $p$ or $r$ is varied, the resonant wavelength changes slightly, as does the transmittance. The resonant wavelength related to the suppressed transmission is redshifted when increasing both $p$ and $r$.

Like the result for the reflectance of the surfacecorrugated metal film $[\underline{6}-\underline{8}, 13]$, the strongly suppressed transmission is related to the resonance anomaly (one of Wood's anomalies), which is due to the excitation of the SPP resonance along the surface. The periodic structure of the gold disk array enables light to couple to the SPPs via reciprocal wave vectors [14]. The SPP resonance appears when the wave vector of the surface plasmon matches the wave vectors of the incident photon and grating as in $k_{\mathrm{sp}}=k_{\|} \pm n G_{x} \pm m G_{y}$, where $k_{\mathrm{sp}}$ is the surface-plasmon wave vector, $k_{\|}$is the component of the incident wave vector in the plane of the grating, and $G_{x}=G_{y}=2 \pi / p$ is the grating's reciprocal vector for the square array. For an ultrathin film with a thickness, $t$, of $15 \mathrm{~nm}$, the SPP modes guided by the two interfaces strongly hybridize owing to the overlap of their fields inside the metal. From Maxwell's equations and boundary conditions, the dispersion relation for the ultrathin film can be derived for these coupled modes [15]:

$$
\tanh \left(\alpha_{m} t\right)=-\frac{\varepsilon_{m} \alpha_{m}\left(\varepsilon_{d 1} \alpha_{d 2}+\varepsilon_{d 2} \alpha_{d 1}\right)}{\varepsilon_{d 1} \varepsilon_{d 2} \alpha_{m}^{2}+\varepsilon_{m}^{2} \alpha_{d 1} \alpha_{d 2}} .
$$

Here $\alpha_{d 1}^{2}=k_{\mathrm{sp}}^{2}-\varepsilon_{d 1} k_{0}^{2}, \alpha_{m}^{2}=k_{\mathrm{sp}}^{2}-\varepsilon_{m} k_{0}^{2}$, and $\alpha_{d 2}^{2}=k_{\mathrm{sp}}^{2}-$ $\varepsilon_{d 2} k_{0}^{2}$, with $k_{0}$ being the incident photon wave number in a vacuum. For the structures we investigate here, $\varepsilon_{d 1}=$ 1 (air) and $\varepsilon_{d 2}=2.088$ (fused glass), and therefore $\varepsilon_{d 1}<\varepsilon_{d 2}$. For this strongly asymmetric geometry, the
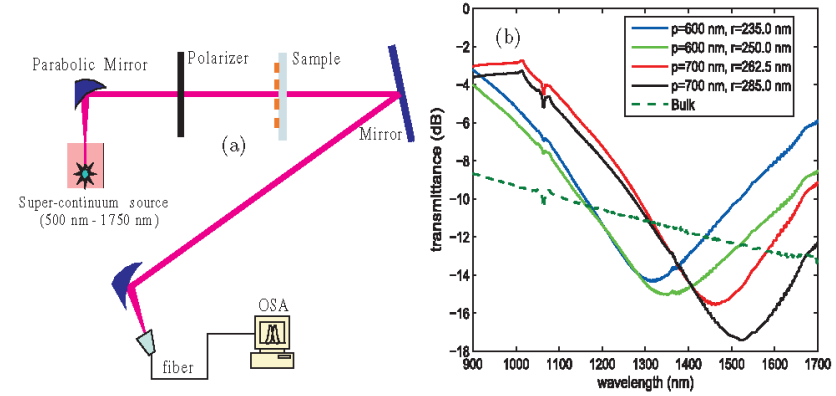

Fig. 2. (Color online) (a) Free-space measurement setup. (b) Measured transmittance spectra for periodic arrays of metal disks (shown by the solid curves) when tuning the structure parameters. As a reference, the dashed curve shows the transmittance spectrum through a metal film with the same thickness as the disks.
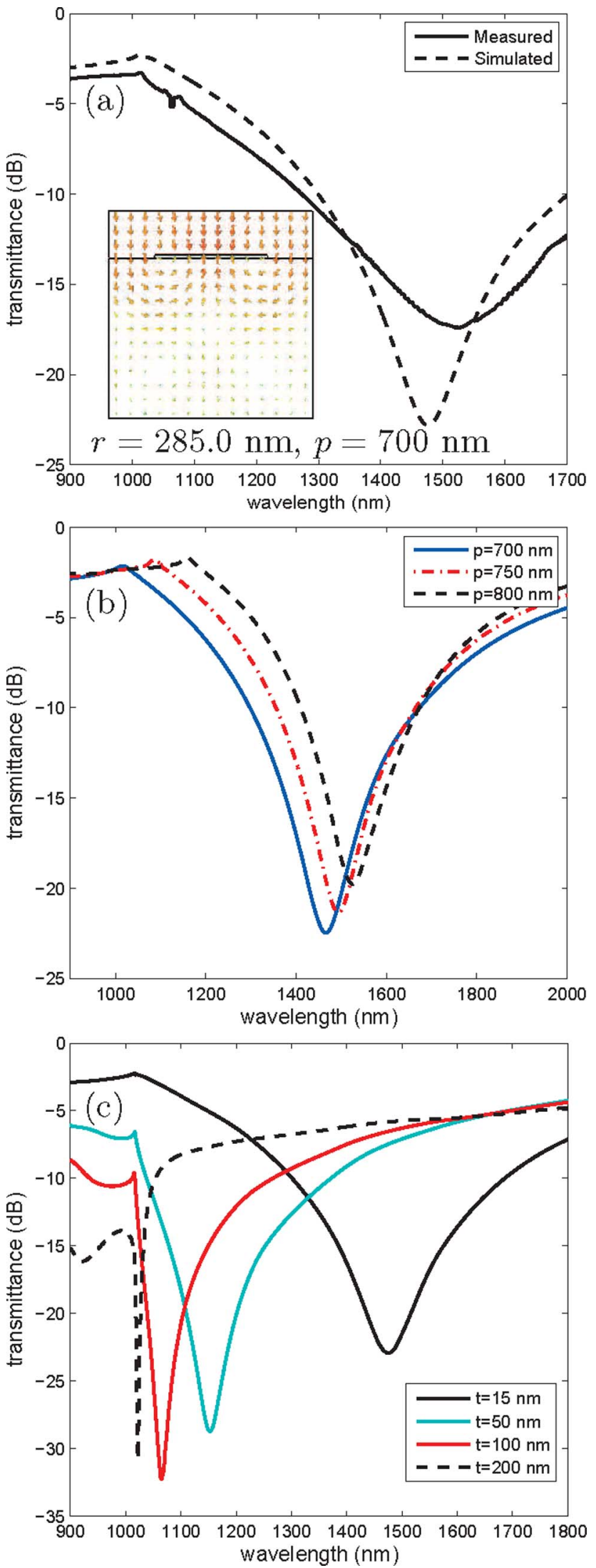

Fig. 3. (Color online) (a) Measured (solid curve) and simulated (dashed curve) transmittance spectra for the case of $t=15 \mathrm{~nm}, r=285 \mathrm{~nm}$, and $p=700 \mathrm{~nm}$. (b) Simulated transmittance spectra as a function of the period $p$. (c) Simulated transmittance spectra as a function of the thickness $t$. 
ultrathin metallic film supports only a single strongly damped short-range surface-plasmon mode. For example, when $\lambda_{0}=1524 \mathrm{~nm}$, the effective refractive index of the SPP mode is around $1.50-0.011 j$. Of course, for an in-plane modulated thin film, Eq. (1) will be subject to the band-folding phenomena associated with the finite value of the reciprocal lattice vector, $G$.

In Fig. 2(b), one observes that cusps occur around $1013 \mathrm{~nm}$, in the case of $p=700 \mathrm{~nm}$, the cusps being independent of the radii of the metal disks. Previous studies $[6,13]$ have shown that the cusps are related to the threshold phenomenon associated with light diffracted parallel to the surfaces, the so-called Rayleigh anomaly (RA), which happens when $\lambda_{\mathrm{RA}}=p \sqrt{\varepsilon_{d}}$. When the wavelength is above the RA point, the in-plane wave vector is always larger than the wave vector of the light, thus indicating that the reflection and transmission of high orders can be negligible. Instead of transferring energy to the high-order diffractions, the transmission of light is suppressed because of the coupling of light into the SPPs along the surface. Obviously, the structure determines the coupling efficiency between the incident wave and the SPP. When the structure is properly designed, the transmission can be totally suppressed. Naturally, the Rayleigh anomaly is determined entirely by the period, $p$, of the structure and the permittivity of the surrounding medium, $\varepsilon_{d}$, while having no dependence on the structure's material and parameters. The wavelength of the $\mathrm{RA}$ related to the $(1,0)$ diffraction order appears at $\lambda_{\mathrm{RA}}=700 \times 1.445=1011.5 \mathrm{~nm}$, showing a good agreement with the wavelength observed from the measurement. We emphasize that the dips around $1065 \mathrm{~nm}$ in Fig. 2(b) are artifacts arising from the supercontinuum light source.

The nontrivial effect, i.e., near-zero transmission, was also verified by numerical simulations (performed with the aid of a commercially available finite integration method [16]). For the optical properties of gold, we used the experimental data from [17]. Figure 3(a) represents the measured (solid curve) and simulated (dashed curve) transmittance spectra when $r=285 \mathrm{~nm}$ and $p=700 \mathrm{~nm}$, illustrating a good agreement. The inset in Fig. 3(a) shows the power flow at the resonance. For a gold disk array at the resonance $\lambda_{0}=1524 \mathrm{~nm}$, the transmittance $(T)$, reflectance $(R)$, and absorption $(A)$ are $0.51 \%$, $88.23 \%$, and $11.26 \%$, respectively. Note that $T=10.21 \%$, $R=85.16 \%$, and $A=4.63 \%$ for the case of the closed film. We emphasize that the excitation of the SPPs results in the suppression of transmission and the increase of absorption as well as reflection. The transmittance as a function of the period is shown in Fig. 3(b). The transmittances are nearly zero at the resonant positions, and the RAs coincide with the predicted spectral position. Figure 3(c) illustrates how the resonances shift when tuning the thickness of the gold disk. The resonances are blueshifted when the thickness is increased. As mentioned above, the SPP modes guided by the two interfaces strongly couple, while they are almost decoupled for a thick film, i.e., $t=200 \mathrm{~nm}$. Under this circumstance, the SPP resonance is related only to the mode for a single interface. From the dispersion of the SPP at an interface between a dielectric and a metal, one knows that the SPP resonance is very close to the RA, as shown by the dashed curve in Fig. 3(c). Meanwhile, the transmittances for all cases are nearly zero at the resonances. Besides, it can be seen in Fig. 3(c) that the RAs are independent of the film thickness.

In summary, we have experimentally demonstrated near-zero transmission of light through gold disk arrays whose thickness is on the order of its skin depth. The experimental results have been verified by numerical simulations, and the suppressed transmission is attributed to the excitation of SPP waves. We have also shown the Rayleigh anomalies, which have the same positions as those predicted theoretically. The suppressed transmission results in enhanced absorption, which is potentially interesting in the context of energy harvesting for solar cells and photocatalysis.

This work is partly supported by the Danish Research Council for Technology and Production Sciences (grant 274-07-0379) and the Catalysis for Sustainable Energy Initiative Center, funded by the Danish Ministry of Science, Technology and Innovation.

\section{References}

1. I. S. Spevak, A. Y. Nikitin, E. V. Bezuglyi, A. Levchenko, and A. V. Kats, Phys. Rev. B 79, 161406 (2009).

2. T. W. Ebbesen, H. J. Lezec, H. F. Ghaemi, T. Thio, and P. A. Wolff, Nature 391, 667 (1998).

3. J. Braun, B. Gompf, G. Kobiela, and M. Dressel, Phys. Rev. Lett. 103, 203901 (2009).

4. S. Xiao, J. Zhang, L. Peng, C. Jeppesen, R. Malureanu, A. Kristensen, and N. A. Mortensen, Appl. Phys. Lett. 97, 071116 (2010).

5. Z. Sun, X. Zuo, and Q. Lin, Plasmonics 5, 13 (2009).

6. R. W. Wood, Philos. Mag. 4, 396 (1902).

7. U. Fano, J. Opt. Soc. Am. A 31, 213 (1941).

8. A. Hessle and A. A. Oliner, Appl. Opt. 4, 1275 (1965).

9. J. A. Porto, F. J. Garcia-Vidal, and P. B. Pendry, Phys. Rev. Lett. 83, 2845 (1999).

10. S. Xiao and M. Qiu, J. Opt. A 9, 348 (2007).

11. F. J. García de Abajo and J. J. Sáenz, Phys. Rev. Lett. 95, 233901 (2005).

12. S. Xiao, L. Peng, and N. A. Mortensen, Opt. Express 18, 6040 (2010).

13. L. O. M. Rayleigh, Philos. Mag. 6, 60 (1907).

14. H. Raeher, Surface Plasmons on Smooth and Rough Surfaces and on Gratings (Springer, 1988).

15. F. Yang, J. R. Sambles, and G. W. Bradberry, Phys. Rev. B 44, 5855 (1991).

16. CST Microwave Studio, CST GmbH, Germany.

17. P. B. Johnson and R. W. Christy, Phys. Rev. B 6, 4370 (1972). 